Streamer knotwilg branching: sudden transition in morphology of positive streamers in highpurity nitrogen

This content has been downloaded from IOPscience. Please scroll down to see the full text. 2015 J. Phys. D: Appl. Phys. 48355202

(http://iopscience.iop.org/0022-3727/48/35/355202)

View the table of contents for this issue, or go to the journal homepage for more

Download details:

IP Address: 131.155.2.68

This content was downloaded on 17/08/2015 at $11: 53$

Please note that terms and conditions apply. 


\title{
Streamer knotwilg branching: sudden transition in morphology of positive streamers in high-purity nitrogen
}

\author{
L C J Heijmans ${ }^{1}$, T T J Clevis ${ }^{1}$, S Nijdam ${ }^{1}$, E M van Veldhuizen ${ }^{1}$ and \\ U Ebert ${ }^{1,2}$ \\ ${ }^{1}$ Department of Applied Physics, Eindhoven University of Technology, P.O. Box 513, 5600 MB \\ Eindhoven, The Netherlands \\ 2 Centrum Wiskunde and Informatica (CWI), PO Box 94079, 1090 GB Amsterdam, The Netherlands \\ E-mail: 1.c.j.heijmans@tue.nl and s.nijdam@tue.nl
}

Received 21 April 2015, revised 18 June 2015

Accepted for publication 30 June 2015

Published 5 August 2015

\begin{abstract}
We describe a peculiar branching phenomenon in positive repetitive streamer discharges in high purity nitrogen. We name it knotwilg branching after the Dutch word for a pollard willow tree. In a knotwilg branching a thick streamer suddenly splits into many thin streamers. Under some conditions this happens for all streamers in a discharge at about the same distance from the high-voltage electrode tip. At this distance, the thick streamers suddenly bend sharply and appear to propagate over a virtual surface surrounding the high-voltage electrode, rather than following the background electric field lines. From these bent thick streamers many, much thinner, streamers emerge that roughly follow the background electric field lines, creating the characteristic knotwilg branching. We have only found this particular morphology in high purity nitrogen at pressures in the range 50 to $200 \mathrm{mbar}$ and for pulse repetition rates above $1 \mathrm{~Hz}$; the experiments were performed for an electrode distance of $16 \mathrm{~cm}$ and for fast voltage pulses of 20 or $30 \mathrm{kV}$. These observations clearly disagree with common knowledge on streamer propagation. We have analyzed the data of several tens of thousands of discharges to clarify the phenomena. We also present some thoughts on how the ionization of the previous discharges could concentrate into some pre-ionization region near the needle electrode and create the knotwilg morphology, but we present no final explanation.
\end{abstract}

Keywords: Streamer, Streamer branching, Streamer discharge morphology, Leftover ionisation, Knotwilg

(Some figures may appear in colour only in the online journal)

\section{Introduction}

A streamer discharge may form when a sufficiently large electric voltage is applied to a gas [1]. Streamers are ionised fingers that initiate in a region with a high electric field. The streamer tip enhances the electric field in front due to its charge layer. With this electric field enhancement the streamers can propagate into regions where the background electric field is below the breakdown field. Streamers create the conducting path of sparks [2,3] and of lightning leaders within and around thunderclouds $[4,5]$, and they can be seen directly as huge sprite discharges in the thin air high above thunderclouds $[6,7]$. In a streamer discharge, electrons are accelerated and deposit energy in the gas in the form of excitations and ionization in a process far from equilibrium, allowing for very efficient chemical reactions while the gas stays cold, i.e. there is little energy deposited in the kinetic energy of molecules. Streamers are applied, for example, in gas and water cleaning [8-11], ozone generation [9], particle charging [9, 12] and flow control [13, 14].

In the present work we concentrate on a very special streamer morphology, the knotwilg branching. The word 


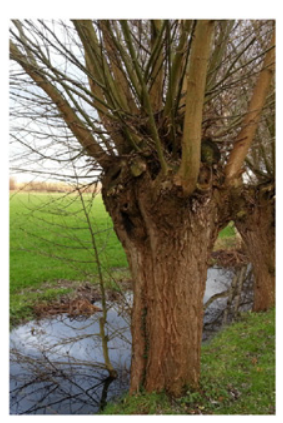

(a)

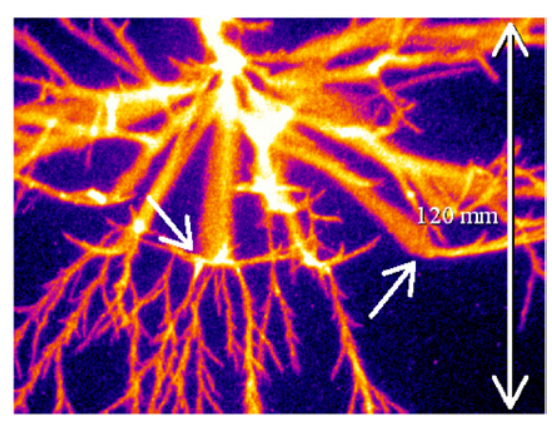

(b)
Figure 1. (a) A pollard willow tree (in Dutch: knotwilg). (b) Image of a discharge at $2 \mathrm{~Hz}$ repetition rate with a $20 \mathrm{kV}$ positive pulse from the C-supply in a vessel of 100 mbar of high purity nitrogen. The exposure time was limited to roughly the first $200 \mathrm{~ns}$ of the discharge. Examples of the knotwilg branching are indicated by arrows.

'knotwilg' is the Dutch name for a pollard willow tree with a thick stem and many thin branches (figure 1(a)); it is formed through regular harvesting of the outer branches. In a knotwilg streamer, as shown in figure 1(b), thick streamer channels suddenly transit into many, much thinner streamer channels. The knotwilg structures have been shortly mentioned by Clevis et al [15], but have not been analyzed further. Though we have found this strange morphology only in a very specific parameter regime up to now, namely for positive streamers in high purity nitrogen of about 100 mbar at pulse repetition frequencies of about $1 \mathrm{~Hz}$, we believe that these structures are so unexpected and against common wisdom of streamer motion, that they teach us about the limitations of current rules of thumb of streamer motion and challenge us to find a physical explanation. We will contribute to this search for theory in this paper as far as we have come. But the main topic of the paper is a very careful experimental examination of the phenomenon.

In the next section, we will introduce the experimental setup, that was used for this research. Section 3 further introduces the knotwilg structures with some general observations on their appearance. Thereafter the effects of the voltage pulse on the knotwilg branchings are shown in section 4 . Section 5 reports on the velocity of the streamer and the influence the knotwilg branching has on it. In section 6 observations are reported of different streamer discharges showing streamers following virtual surfaces. This is then all finally used in section 7 , where a hypothesis explaining the observed effects is given.

\section{Setup}

Streamers can have positive and negative polarity. Only positive streamers are discussed in this paper, i.e. those emerging from a positively charged electrode. These are the focus of most streamer applications and investigations, because they are the easiest to create [16].

The discharge setup employed in this work is the same as previously used by Nijdam et al [17]. Figure 2(a) depicts a worked-open schematic drawing of the vacuum vessel with the discharge geometry. The electrode configuration

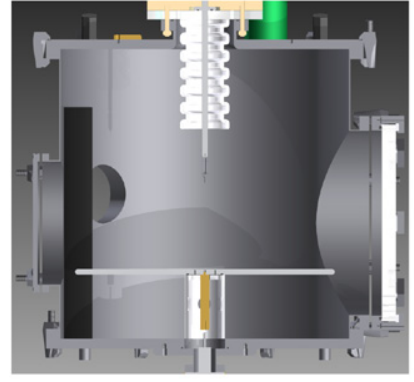

(a)

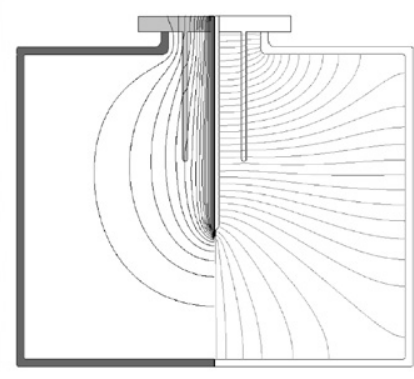

(b)
Figure 2. (a) Worked-open drawing of the used vessel with the electrode configuration inside. (b) Schematic drawing of (left half) the electric equipotential lines and (right half) the electric field lines in the used point-plane setup.

has a point-plane geometry. A sharp tip is positioned at the top. It has a curvature of about $70 \mu \mathrm{m}$ and is used as anode. A grounded plate is located $160 \mathrm{~mm}$ below. This is used as cathode. Figure 2(b) illustrates the equipotential lines and field lines of the background electric field in a simplified vessel when a high voltage is applied to the tip. It shows a high electric field around the tip.

The present measurements are all performed in 100 mbar nitrogen 6.0, i.e. in nitrogen with less than 1 ppm contaminations. The leakage and out-gassing of the vessel is measured. This is found to also cause less than 1 ppm contaminations. This is important, because small contaminations have been shown to influence the streamer morphology [17].

Most of the presented measurements use the so-called C-supply. This uses a discharging $2 \mathrm{nF}$ capacitor to create the voltage pulse, as described by Briels et al [18]. A typical voltage pulse is shown in figure 3 . It has a rise time of tens of nanoseconds and a decay time of around $10 \mu \mathrm{s}$. This time is much shorter than the typical time between pulses which is at least $100 \mathrm{~ms}$.

The streamers are imaged using a Stanford Computer Optics 4 Quick E intensified CCD (ICCD) camera. It can be used to image either a complete discharge or a part of the streamer propagation by modifying the exposure time, because only the head of a streamer emits light. Note that for each images presented in this paper, the colour has been optimised for clarity. Therefore intensities between different images can not be directly compared.

\section{The phenomenon of Knotwilg branching}

In our setup, knotwilg branching has been found in discharges in high purity nitrogen at repetition rates of around $1 \mathrm{~Hz}$ and higher for pressures between 50 and 200 mbar. As they appear most prominently at 100 mbar, all discharges shown in the remainder of this paper will be in 100 mbar nitrogen 6.0, unless specifically stated otherwise.

Figure 4 shows time resolved images of the streamer development at a repetition frequency of $2 \mathrm{~Hz}$. For these images, the camera gate is always opened before the start of the voltage pulse, while the closing time is varied. This delay after the start of the voltage pulse is indicated in each of the images. 


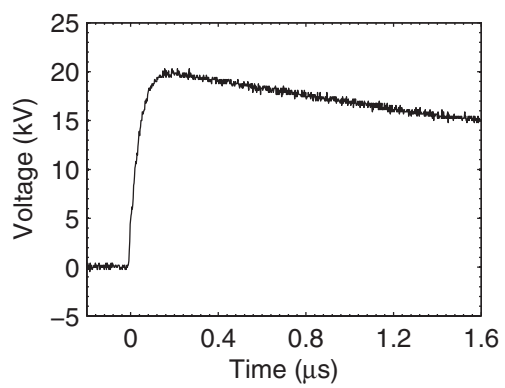

Figure 3. Measurement of typical voltage pulse created with the C-supply.
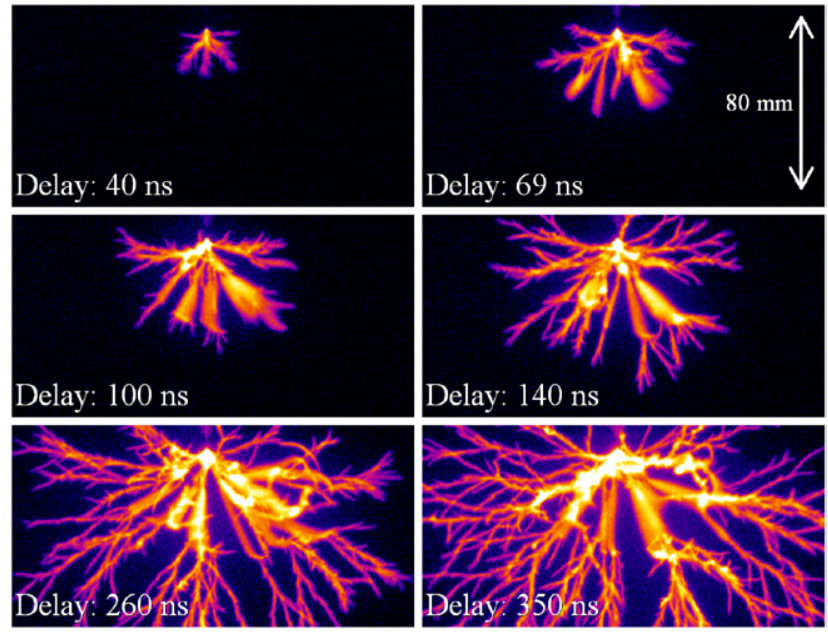

Figure 4. Images of the streamer development. The trajectory of the streamer tip from the discharge start to the indicated time can be seen. The discharges were created with $20 \mathrm{kV}$ pulses from the C-supply at $2 \mathrm{~Hz}$ repetition rate in 100 mbar pure nitrogen. The total gap is $160 \mathrm{~mm}$. Note that each image is from a different discharge.

Note that the camera does not allow to take multiple images of one streamer discharge. Therefore the shown images are all from different discharges under the same conditions.

The figure shows how the streamers emerge from the tip and propagate as channels with increasing width and little branching until knotwilg branching occurs after 70 to 80 ns. Here the thick streamers split into many much thinner streamers. Then the thinner streamers continuously branch and propagate. After about $320 \mathrm{~ns}$, they reach the cathode plate. Later the streamers continue to fill the gap until the end of the voltage pulse.

\subsection{The role of the discharge repetition frequency}

Figure 5 illustrates how the knotwilg structure is built up by the previous discharges in a repetitive mode. After a pause of 1 min without discharges, a series of discharges at repetition rate of $2 \mathrm{~Hz}$ (left column) and $4 \mathrm{~Hz}$ (right column) was performed. Out of these series, the first, second, fifth and tenth discharge are shown.

It can be seen that the first discharge has no morphological peculiarities, but that the remainders of the previous discharges change the structure of the subsequent discharges,
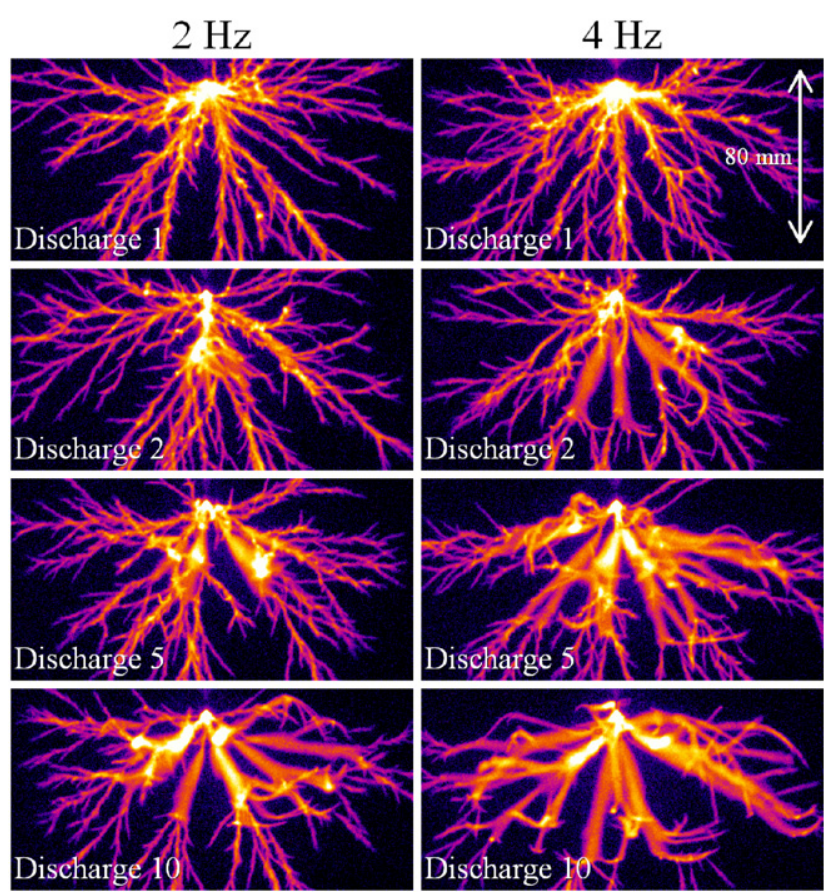

Figure 5. Discharge (from top to bottom) one, two, five and ten of a discharge series at (from left to right) 2 and $4 \mathrm{~Hz}$ repetition rate. Other conditions are equal to those in figure 4 . There was a pause of about a minute of no discharges before the first discharge.

until the knotwilg structure appears. In the $4 \mathrm{~Hz}$ case, the clear knotwilg structure appears already in the second discharge. In the $2 \mathrm{~Hz}$ case, some indications of knotwilg formation are visible in the second discharge of the series, and the structure is clearly developed in the fifth discharge.

We have searched for the phenomenon at repetition frequencies from 0.1 to $20 \mathrm{~Hz}$. Knotwilg structures were found in discharges in 100 mbar nitrogen 6.0 for repetition rates around $1 \mathrm{~Hz}$ and higher.

3.1.1. Position dependence. The knotwilg branchings all seem to form on a spherical shell around the high-voltage electrode. Inside the shell, the streamers are thick, whereas outside thin streamers appear. As leftover electrons have been found to locally enhance streamer thickness [15, 19], one might thus expect that the leftover electrons only appear in a sphere surrounding the high-voltage electrode. This is however unlikely for two reasons.

Firstly, the complete discharge is much bigger than the length of the thick streamers before the knotwilg structures. It fills the entire discharge gap. The created electron density may be estimated by looking at the light emission during a discharge. This has been measured as function of the distance from the tip. No steep gradients are found in the first discharges, instead a smooth intensity decay is seen in the region that shows the knotwilg structures in the next discharge.

It is possible that the electrons drift between the discharges. This might e.g. be caused by a small electric field caused by the end of the voltage pulse. This option requires a more extensive study to investigate the post-discharge dynamics, including the electron recombination and attachment, to see 
wether such a drift is feasible and could cause an increased electron density region.

Secondly, Nijdam et al [20] calculated the diffusion of ions and electrons from a streamer channel. This is in the order of centimetres in the $0.5 \mathrm{~s}$ between two discharges at $2 \mathrm{~Hz}$. This would smoothen out any steep gradient. They confirmed this by showing that streamers in subsequent discharges do not show a preference to follow the same path for repetition rates up to $10 \mathrm{~Hz}$.

In the current research, the path repetition measurements have been repeated and extended up to $20 \mathrm{~Hz}$. Subsequent streamers start following the same path at around $14 \mathrm{~Hz}$ and higher. At these repetition rates, the streamers are still influenced by the location of the leftover electrons from the previous discharge. This is at timescales an order of magnitude smaller than when knotwilg structures are first observed. Also, this is at length scales of the size of the individual streamers, as opposed to the entire spherical region around the tip.

In [21] streamer discharges were created in 67 and 133 mbar pure nitrogen by two pulses with a variable delay. For short delays, subsequent streamers follow the same path; this stops at a delay between 10 and $40 \mathrm{~ms}$. This time scale is of the same order of magnitude as the path-following in the present measurements. The slightly shorter time might be due to the leftover electrons being only created by one previous discharge, whereas with the higher repetition rate it is an accumulation of all previous discharges.

Although the knotwilg structures indicate a strong boundary, no evidence was found for a steep gradient in the electron density. Leftover electrons should also appear beyond the knotwilg position with no indications of sharp transitions. Furthermore, diffusion will smoothen out any steep density gradients.

An alternative explanation might be a critical electron density. The streamers might follow the leftover ionisation at precisely this level. This would rule out the requirement for a steep gradient. The authors could however not find any indications of a critical electron density in streamer discharges in literature, either experimentally or theoretically. A critical leftover electron density has recently been found by Carbone and Nijdam [22] in a different plasma discharge (a pulsed microwave discharge in argon). This caused the discharge morphology to change completely on a small change in repetition frequency.

\subsection{Effect of oxygen concentration}

We have observed gas purity to be important for the appearance of knotwilg structures. They are suppressed by small oxygen contaminations. No knotwilg structures could be found in gas mixtures with an oxygen concentration one or two orders of magnitude above the used 1 ppm contaminations. No exact critical oxygen concentration could be determined. The disappearance of the knotwilg structures might be due to either photo-ionisation or electron attachment.

Photo-ionisation is an important source of electrons for positive streamers in air $[17,23]$. This ionisation is (almost) absent in high purity nitrogen. A larger amount of photo-ionisation will decrease the relative importance of leftover background electrons and may therefore suppress the knotwilg structures.

Electrons can attach to oxygen molecules forming negative ions. In 100 mbar nitrogen with 1 ppm oxygen, the leading reaction for this is $\mathrm{e}^{-}+\mathrm{O}_{2}+\mathrm{N}_{2} \rightarrow \mathrm{O}_{2}^{-}+\mathrm{N}_{2}$ with a reaction rate of $8 \cdot 10^{-32} \mathrm{~cm}^{6} \mathrm{~s}^{-1}$ [24]. This leads to a typical time constant of $2 \mathrm{~s}$, which is much longer than other electron loss reactions [21]. Hence the density of negative oxygen ions is relatively small. Moreover, according to our calculations the density of free electrons is at least two orders of magnitude larger for all relevant situations in this article. Therefore electron attachment and detachment can be neglected-except if the different mobilities of electrons and ions would lead to local enhancements of the density ratio.

\section{Voltage pulse}

Briels et al [16] have shown that the applied voltage, and thus electric field, has a large effect on streamer thickness in ambient air. Grabowski et al [25] explained a sudden change from thick to thin streamers by a large drop in voltage. Similarly, Clevis et al [15] found knotwilg structures, when using a block-shaped voltage pulse with a large voltage drop. No such effect has however been found for the present knotwilg structures.

Figure 6(a) shows the measured voltage for discharges similar to those in figure 5. The blue lines show voltage pulses under the same conditions, but with the vessel at vacuum. For the other lines the current drawn by the starting streamers reduces the peak voltage. The first discharges show a later deviation. This is due to a larger inception delay. It has previously been found that inception delay can be long for a first streamer in fresh gas [20]. This is because there are less background electrons to create the first avalanche.

In the figure, the time between 70 and $80 \mathrm{~ns}$ is indicated in grey. This is when the knotwilg structures appear. In this region the voltage decreases slightly, but there are no steep gradients that can explain the sudden knotwilg structures.

\subsection{Different voltage}

Figures 6(b) and (c) show two voltage pulses, where the C-supply has been modified by replacing the series resistor with a metal wire. This causes a faster voltage rise and oscillations around the start of the voltage pulse. The two pulses differ in the applied voltage. Streamer discharges have been created using both pulses at a repetition rate of $2 \mathrm{~Hz}$. The first discharges have been disregarded, as they were different from subsequent discharges. Time resolved discharge images are shown in figure 7 for the two pulses. Both show the knotwilg branching.

The $30 \mathrm{kV}$ pulses cause thicker streamers that propagate faster (see also section 5). This is in line with measurements by Briels et al [16] for streamers in ambient air. The change in velocity has more influence on the time at which the knotwilg structures appear than on the position at which they appear. Whereas there is roughly a factor two difference in the timing, the position shifts only slightly. Therefore, the knotwilg 


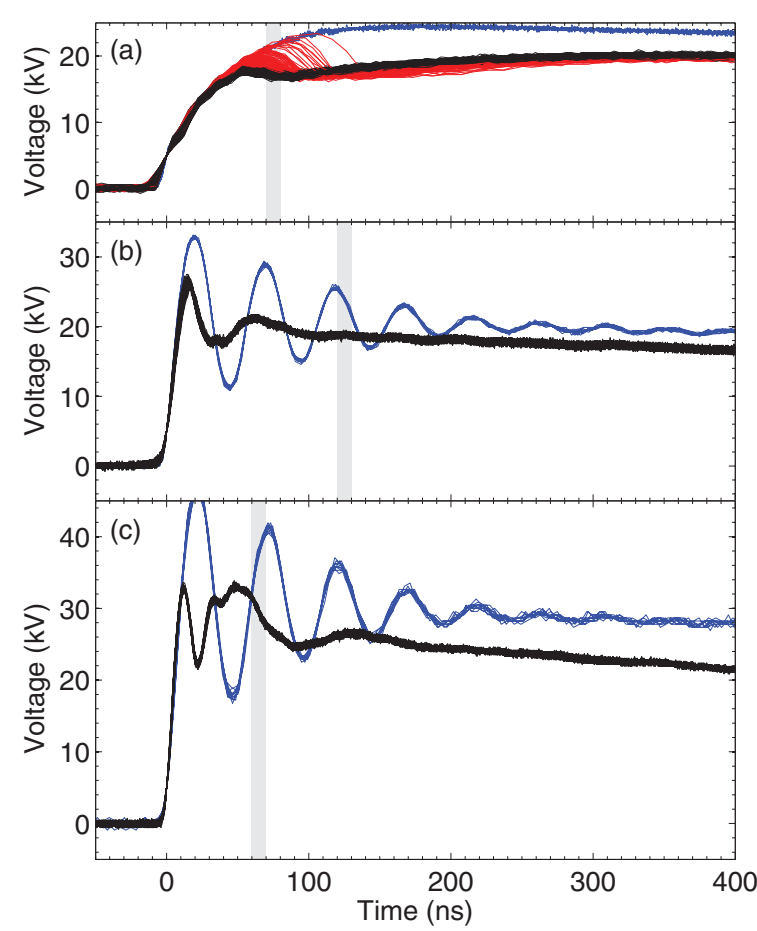

Figure 6. Voltage pulses for the first (red lines) and later (black lines) discharges at $2 \mathrm{~Hz}$ repetition rate in $100 \mathrm{mbar}$ nitrogen 6.0 at voltage of ((a), (b)) $20 \mathrm{kV}$ and (c) $30 \mathrm{kV}$ from the C-supply. In ((b), (c)) a resistor is removed from the circuit. The blue line indicates the voltage pulse when the vessel is at vacuum (pressure $<10^{-6}$ mbar) and no discharge occurs. The time between (a) 70 and 80 ns, (b) 120 and $130 \mathrm{~ns}$ and (c) 60 and $70 \mathrm{~ns}$, where the knotwilg structures appear, is indicated in grey.

branching more likely appears at a certain position, than at a certain time.

The knotwilg position differs less between these two discharge conditions than when compared to the discharges in figure 4. Recall that these discharges had a voltage of $20 \mathrm{kV}$, like those in figure 7(a), but had a longer rise time. Therefore the knotwilg structure appears not as much influenced by the average voltage, but more by smaller features as for example the voltage rise time or the overshoot and oscillations. Clevis et al [15] showed that a slight change in the voltage rise time can have a measurable influence on the streamer length. This influence was explained by the size of the inception cloud, which, on average, propagates slower than streamers. In the present research no inception cloud is directly visible. It is however possible that the voltage rise time has some effect that is important for the knotwilg formation.

Again, note that no steep gradients in the voltage are visible when the knotwilg structures appear. These times are indicated in grey in figure 6.

\section{Velocity}

The knotwilg branching largely changes the morphology. This can be expected to influence the streamer propagation. Therefore we study the streamer velocity in these discharges. This is done using a methode that is previously used by Clevis et al [15] and improved by Chen et al [26].
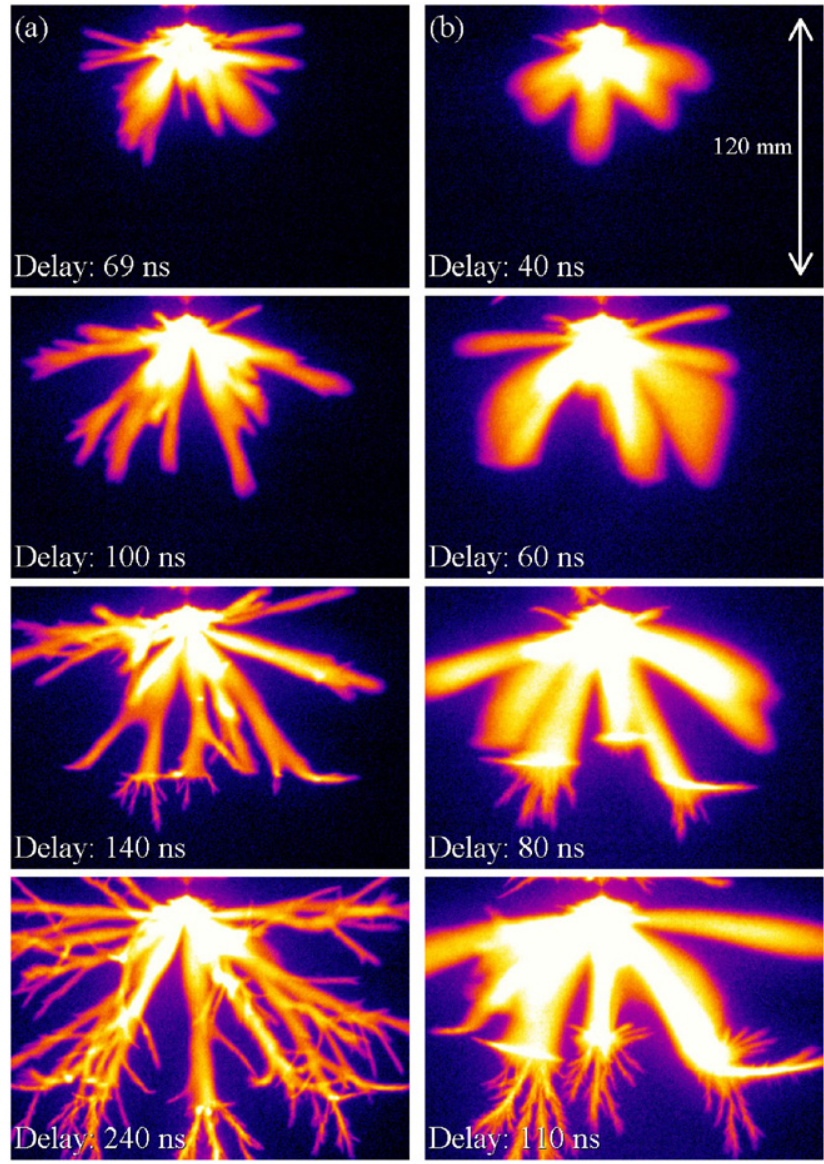

Figure 7. Images of the streamer development at $2 \mathrm{~Hz}$ repetition rate in nitrogen 6.0 as in figure 4, ((a); left column) with the $20 \mathrm{kV}$ voltage pulse shown in figure $6(\mathrm{~b})$ and ((b); right column) with the $30 \mathrm{kV}$ voltage pulse shown in figure 6(c).

The streamer velocity is determined by measuring the streamer length as a function of time. The streamer length is automatically computed from images, like those shown in figures 4 and 7 . This is done in each image by finding the pixels furthest away from the high voltage electrode, on which a streamer is still visible. Their distance from the electrode is taken as the streamer length. This is repeated for hundreds of different streamer discharges under equal conditions, because the short timescales make it impossible to take multiple pictures of one discharge. The result is shown in figure 8 , where the streamer length is plotted as function of time for the three different voltage pulses.

The points at $0 \mathrm{~mm}$ are from discharges that have not yet initiated. This inception can take up to $80 \mathrm{~ns}$ for the first discharge in a series. The discharges with the longest inception delay correspond to the latest voltage deviations in figure 6(a). As discussed above, inception happens faster in subsequent discharges. The first discharges in a series for the other two voltage pulse steps are not shown. Therefore long inception delays are not visible for these in the plots.

The numerous points around $160 \mathrm{~mm}$ indicate streamers that have reached the cathode plate. The streamer 'jumps' to the cathode plate when it is near, due to the high electric field between the streamer tip and the cathode plate and possible secondary electron emission from the plate [15]. 


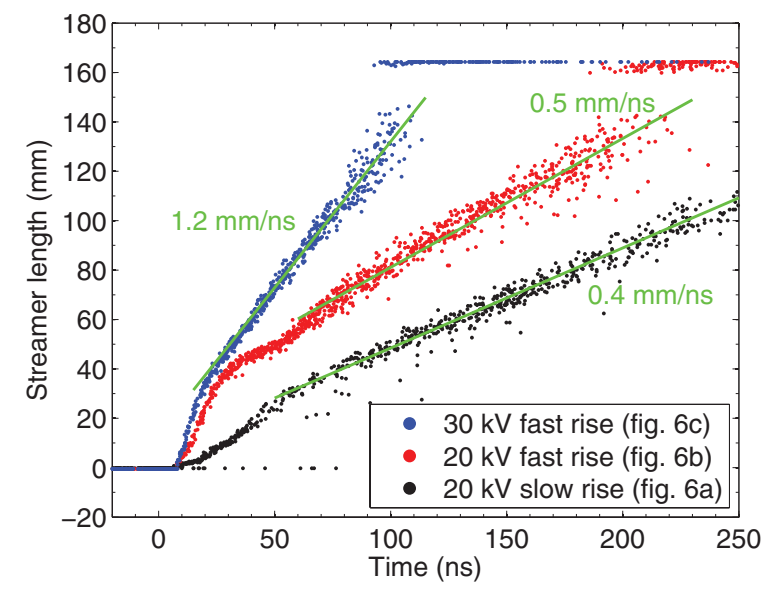

Figure 8. Streamer length as function of time for streamers as imaged in (blue dots) figure 7(b) (with the voltage pulse as in figure 6(c)), (red dots) figure 7(a) (with the voltage pulse as in figure 6(b)) and (black dots) figure 4 (with the voltage pulse as in figure 6(a)). The green lines are added to indicate the average streamer velocity. The plot summarises 2400 discharges.

In the figure it is clear that the streamers at $30 \mathrm{kV}$ propagate faster than those at $20 \mathrm{kV}\left(1.2 \mathrm{~mm} \mathrm{~ns}^{-1}\right.$ versus $0.5 \mathrm{~mm}$ $\mathrm{ns}^{-1}$ (after the bend at $60 \mathrm{~ns}$ )). Briels et al [16] have shown that an increase in background electric field, caused by the higher applied voltage, makes streamers propagate faster. The slow rise streamers are slower than those from the fast rising voltage pulses. This appears mainly in the first $50 \mathrm{~ns}$, where the voltage in this pulse is still rising and thus lower than in the other two pulses. This is similar to what has been found by Chen et al [26].

Below we will first shortly look at the velocity changes appearing in the graph. Thereafter we will investigate the velocity spread, which appears to have a physical background related to the knotwilg branching.

\subsection{Velocity changes}

Figure 8 shows some changes in velocity during the first $50 \mathrm{~ns}$. The streamer velocity can be calculated by taking the derivative of the points in the figure. For a clear result, a smoothing function was first applied. The result is shown in figure 9. Additionally the voltage pulses are shown in this figure.

The velocity and voltage shapes show similar features. This is especially clear in figure 9(b). A voltage dip appears between 20 and $50 \mathrm{~ns}$. This is also visible as a dip in the velocity. The relation is less clear in figures 9 (a) and 9(c). Both figures show the decay in both velocity and voltage after the initial peak. Thereafter the velocity is not directly following the voltage pulse. The tip voltage can thus explain some features of the streamer velocity. However, other aspects also need to be taken into account to explain the complete velocity development.

Remarkably, the formation of the knotwilg branches do not appear to have an influence on the average streamer velocity. No significant change in velocity can be seen around the time when the knotwilg structures appear. Although the streamer diameter decreases significantly, the velocity remains the same.

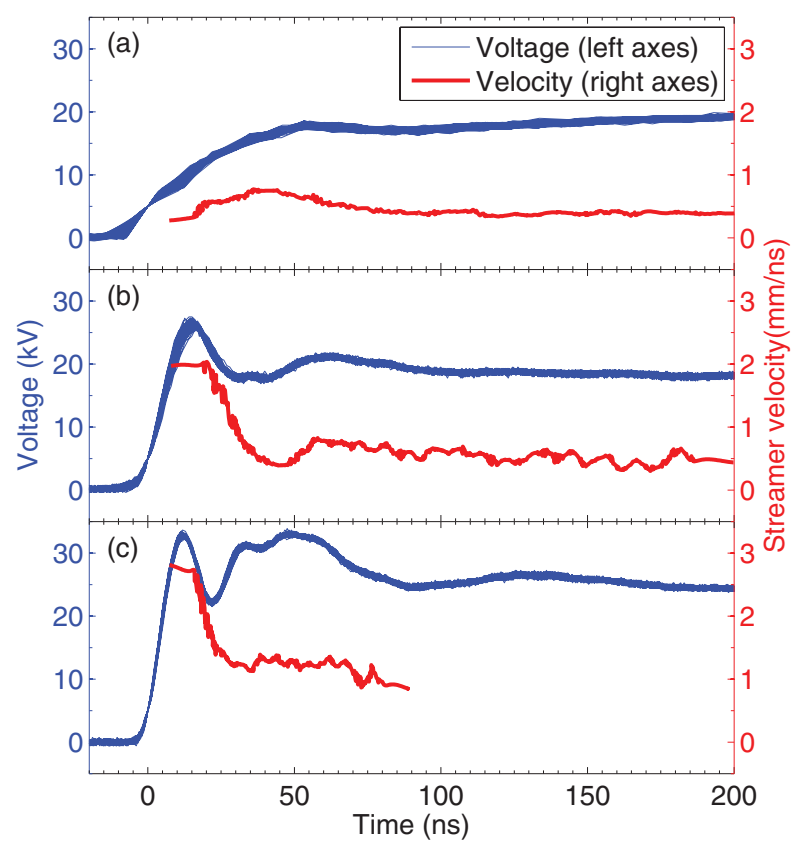

Figure 9. Voltage pulses (blue lines) and streamer velocity (red lines). The discharges are created at $2 \mathrm{~Hz}$ repetition rate in 100 mbar nitrogen 6.0 with the voltage pulse shown in (a) figure 6(a), (b) figure 6(b), (c) figure 6(c).

\subsection{Velocity spread}

As was seen above, there is some spread in the streamer velocities, even under the same conditions. This spread is not purely due to measurement inaccuracies, but also has some physical origin. This is clear when comparing the two columns in figure 10. It shows some of the discharges created with the $20 \mathrm{kV}$ fast rise pulse at $2 \mathrm{~Hz}$. The discharges were selected for having a relatively large deviation from the average length (see also figure 12(b)).The left column contains images of three relatively fast streamers, while the right column contains images of three relatively slow streamers.

The two columns clearly show differences in morphology, although all were created under equal conditions. The slower streamers show the knotwilg branching closer to the tip and earlier in time than the faster streamers. In fact, the faster streamers propagate beyond the imaged region, before showing the knotwilg branching.

Figure 10 also shows feather-like streamers near the tip for the slow streamers [27]. All knotwilg streamers show this feather-like stage close to the tip. It is however much bigger for the relatively slow streamers.

Figure 11 illustrates the three stages in the development of a knotwilg streamer. It starts near the electrode with the feather-like streamers, which change into thick, smooth streamers (figure 11(b)). These show the knotwilg branching as a transformation to thin, branching streamers (figure 11(c)).

Figure 12 illustrates the relation between the velocity and the different stages. Here the streamer length is plotted against time, as done above. The different streamer stages are indicated with colours. Streamers that are still in the first, feather like, stage near the tip are indicated in red; streamers in the second, smooth streamer, stage are indicated in green and streamers 

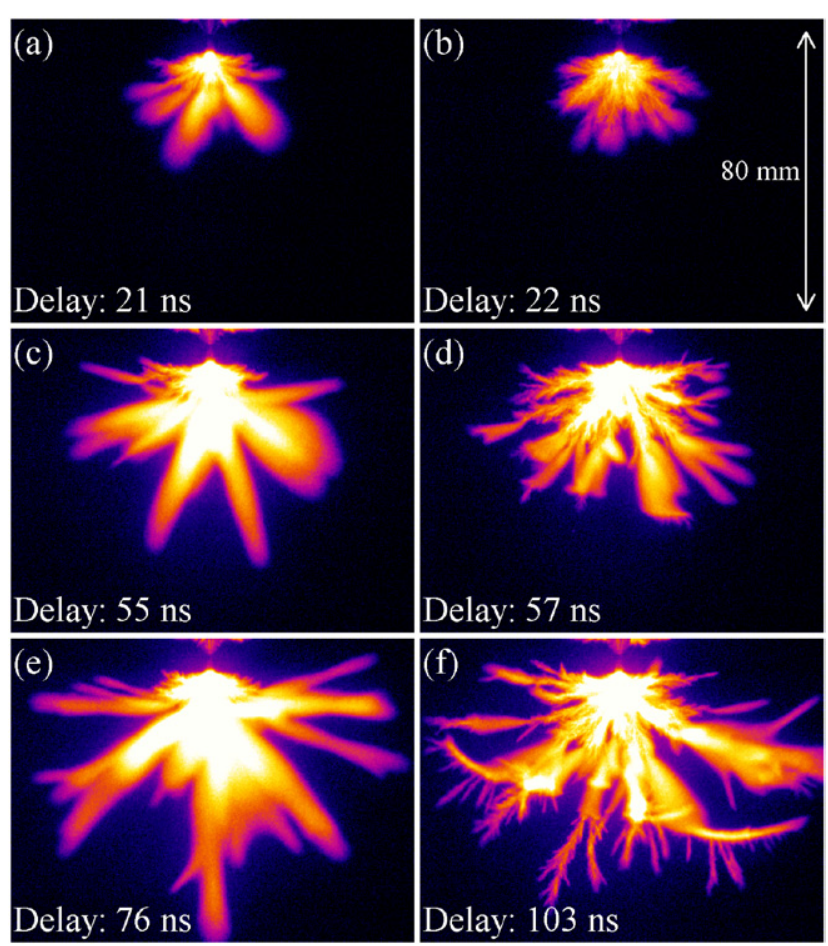

Figure 10. Images of the streamer development as in figure 7(a). The left column contains images of relatively fast streamers, while the right column contains images of relatively slow streamers (see also figure 12(b)). The images show $80 \mathrm{~mm}$ out of the gap length of $160 \mathrm{~mm}$.

in the thin stage after the knotwilg branching are indicated in blue. Figure 12(b) shows the same, but obtained from close-up images. These images are similar to the ones in figure 10; those six discharges are indicated by the stars. Both graphs show that, at a $2 \mathrm{~Hz}$ repetition rate, relatively slow streamers show the knotwilg branching earlier in time than the faster ones.

Figure 13 also shows the velocity development during the different stages, but for three different repetition rates. These can not be directly compared to the measurement in figure 12 , because they were obtained using a slightly different voltage pulse. A slight change in voltage pulse can, as discussed above, have significant effect on the knotwilg branching. The voltage pulse was the same for the three graphs in figure 13; therefore these can be compared.

The length development is shown for repetition rates of 2 , 4 and $8 \mathrm{~Hz}$. The $2 \mathrm{~Hz}$ discharges show a similar trend as above of knotwilg branches appearing closer to the tip and earlier in time for relatively slow streamers. The $8 \mathrm{~Hz}$ discharges however show an opposite behaviour. Here the relatively fast streamers show the knotwilg branching closer to the tip and earlier in time.

An explanation might be found in the amount of background electrons. It has been suggested that a certain optimal level of background electrons exists, which leads to the highest propagation velocity $[21,28]$. A possible handwaving explanation is as follows: with less background electrons, the streamer propagation velocity would be limited, because too few free electrons are available; while with more background electrons, the abundance of electrons would lead to a lower electric field enhancement and thus to a lower propagation velocity.

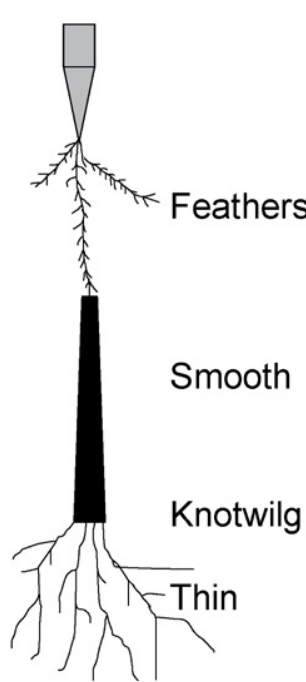

(a) (b)

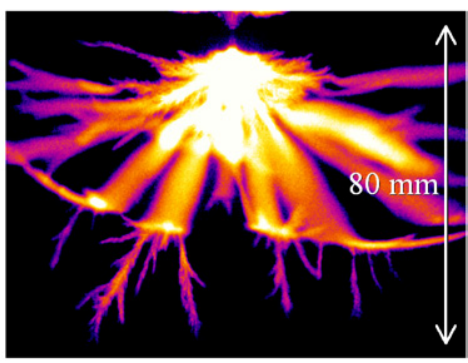

(c)

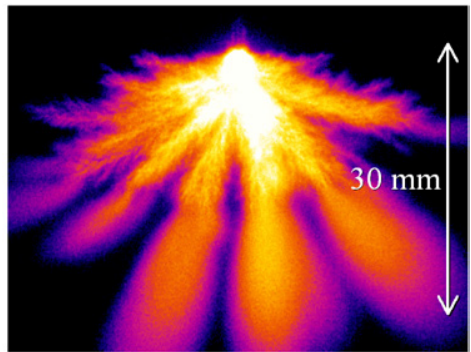

Figure 11. (a) Schematic representation of the three stages in a streamer showing the knotwilg branching. (b) Zoomed-in image of a streamer near the electrode showing the first and transition to the second stage, as depicted in (a). (c) Image of a streamer showing the knotwilg branching as transition from the second to the third stage as depicted in (a).

If $2 \mathrm{~Hz}$ is below this optimum and $8 \mathrm{~Hz}$ is above it, streamers at both repetition rates might behave differently in velocity, but similar to knotwilg branching on leftover ionisation fluctuations. If, for some reason, the ionisation in one particular discharge (i.e. one dot in figure 13) were higher, this would, in the $2 \mathrm{~Hz}$ case, lead to a faster discharge with later knotwilg branching and, in the $8 \mathrm{~Hz}$ case, to a slower discharge with later knotwilg branching. No source could however be found for these ionisation fluctuations. Further measurements are advised to check this hypothesis.

\section{Comparible observations}

A different way of looking at the knotwilg structures is to not see them as a sudden branching of a thick streamer into many thin streamers, but rather as a thick streamer making a sharp bend, wherefrom thin side-branches emerge.

\subsection{Bending streamers}

This explanation is supported by the discharges shown in figure 14. These are created with a lower applied voltage $(10 \mathrm{kV})$ at a higher pressure $(200 \mathrm{mbar})$ in the same highpurity nitrogen. Figure 14 shows a regular discharge at a $1 \mathrm{~Hz}$ repetition rate with streamers propagating from the tip to the cathode plate. At higher repetition rates (above around $4 \mathrm{~Hz}$ ), the streamers no longer propagate to the cathode plate. Instead they suddenly bend sharply, after some distance. 


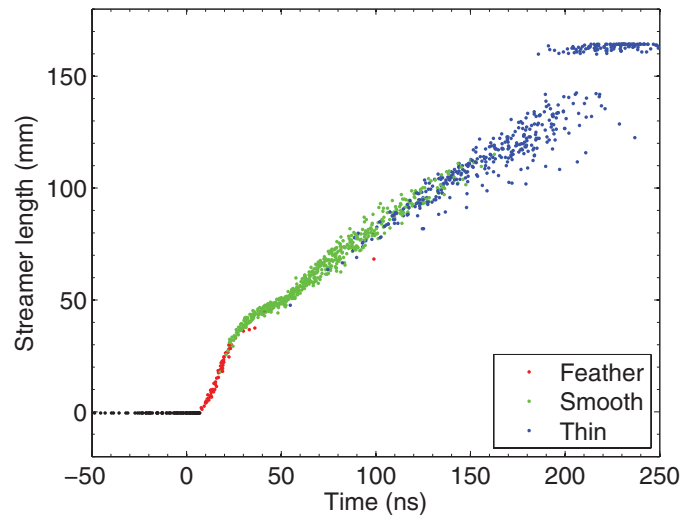

(a)

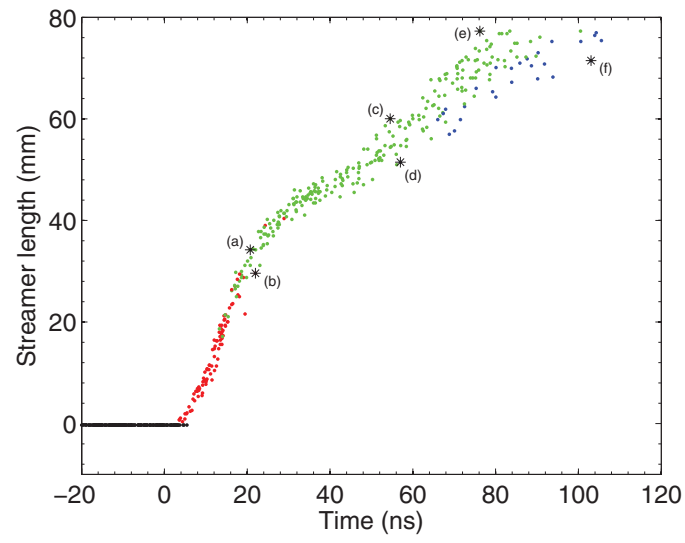

(b)

Figure 12. (a) Graph of the streamer development as in figure 8 for the $20 \mathrm{kV}$ fast rise voltage pulse at repetition rate of $2 \mathrm{~Hz}$.The different stages are indicated using colours. (b) Same, but obtained with the camera positioned closer by; the markers for the discharges in figure 10 are indicated using stars. Both plots summarise 1200 discharges.

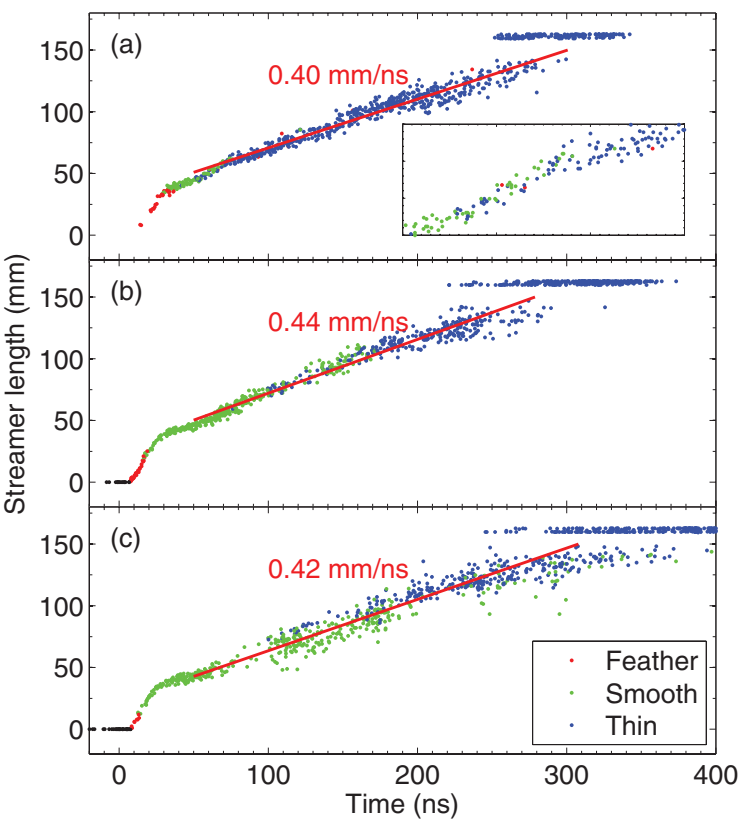

Figure 13. Graph of the streamer development as in figure 12 at repetition rates of (a) $2 \mathrm{~Hz}$, (b) $4 \mathrm{~Hz}$ and (c) $8 \mathrm{~Hz}$. The different stages are indicated using colours. The red lines are added to indicate the average streamer velocity. The inset is a zoom-in of the region around the knotwilg branching. The plots summarise (a) 800 , (b) 950 and (c) 1248 discharges.

This behaviour has similarities with the knotwilg branching. This also shows a sudden morphology change at a certain distance from the tip. It also appears that the thick streamers do not just branch, but rather bend sharply, like in the upward bending streamers. This can be seen for example on the right in figure $1(\mathrm{~b})$.

The discharge in figure 14(b) does not show thin branches toward the cathode plate. This absence might be due to the lower applied voltage compared to the knotwilg branches. Additionally the pressure is higher. This causes an even lower reduced electric field $E / n$. In this case the applied voltage is enough to support the discharge. At lower voltages (at the same pressure) no discharge occurs. When applying a higher

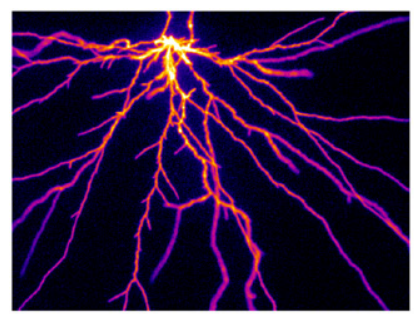

(a)

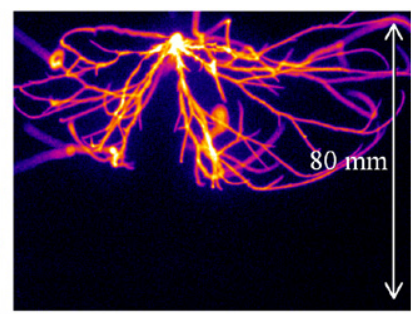

(b)
Figure 14. Image of streamer discharge created with a $10 \mathrm{kV}$ pulse from the C-supply in 200 mbar nitrogen 6.0 with a repetition rate of (a) $1 \mathrm{~Hz}$ and (b) $10 \mathrm{~Hz}$.

voltage to the discharges in figure 14(b), branches propagating to the cathode plate start appearing from the bending streamers.

It thus seems that the knotwilg structures start as bending streamers. The streamers start propagating outward from the tip. After some distance they bend and start following some spherical surface around the tip.

\subsection{Streamers following virtual surfaces}

Nijdam et al [28] documented a peculiar morphology in which streamers follow a virtual surface. It starts with a negative inception cloud. Due to reflections in the pulse forming network, this is directly followed by positive streamers. These do not follow the background electric field lines. Instead they propagate over the surface of the previous inception cloud. This movement is probably due to an interplay between the free electrons inside the surface, attracting the streamers, and the shielding of the electric field enhancement by these electrons, repelling them.

These streamers are similar to the knotwilg branching streamers after they bend. They also appear to follow some virtual surface. However, these discharges have been observed in 600 and 1000 mbar artificial air, and they propagate roughly $100 \mathrm{~ns}$ after the free electrons are created (in the negative inceptor cloud), while the knotwilg branching is observed in 
100 mbar pure nitrogen propagate half a second after the electrons are created (in the previous discharge). In this long pause, the electrons are expected to recombine and diffuse, before the new streamers start. This means that the border between an ionised and non-ionised region has a much less sharp gradient. On the other hand, the streamers from [28] were created at a higher pressure and in air instead of pure nitrogen. Therefore causes different electron losses due to electron attachment to oxygen are much more rapid, and therefore the intrinsic timescales are much shorter.

In the double pulse experiments in [21] streamers in 67 and 133 mbar nitrogen were seen propagating over the edge of the channel that was ionised by a streamer in a previous discharge. This effect was visible with intervals between the streamer discharges up to the order of hundred microseconds. At intervals up to tens of milliseconds, streamers were still seen to follow the paths of previous streamers, but it could no longer be determined whether they followed the edge of the streamer channel.

Again, these experimental timescales are shorter than in the case of the knotwilg branching. But also the length scales are shorter. Here they are of the order of the streamer width, while in the knotwilg branches they are of the size of the large spherical region filling almost half the discharge gap.

In [29] an ionised region was created using a laser. It was shown that streamers in 133 mbar nitrogen can follow the pre-ionised path. It was found that streamers can, in this way, propagate perpendicular to the electric field. This was shown with delays between the laser pulse and the discharge of up to $2 \mathrm{~ms}$. Again, this is shorter than the time between discharges in the present experiment, but the ionized channel is narrower as well.

\section{Summary and conclusions}

A peculiar streamer morphology has been found. It is named knotwilg branching. It was found for streamer discharges from 50 to 200 mbar of nitrogen at pulse repetition rates above $1 \mathrm{~Hz}$. The authors are not aware of observations of similar knotwilg branches in other streamer discharge setups. This is probably due to the very limited parameter regime in which they appear and the required high nitrogen purity. The presently used setup has been specially built for working with high purity gasses. Small amounts of oxygen contamination prevent the knotwilg structures from appearing. Therefore it is unlikely that the knotwilg branches will be observed in a setup containing more impurities.

Experimental measurements were performed to gain insight in the processes that create the peculiar knotwilg structures. We conclude that their appearance is not directly caused by a change in applied voltage. Unfortunately no solid conclusion can yet be drawn on what does cause their appearance. A possible hypothesis is given.

An important aspect is not only the knotwilg branching itself, but also the bending preceding the branching. The thick smooth streamer does not just branch into thin streamers, but rather makes a sharp turn. Similarly bending streamers without the branches were shown in section 6 .
When the applied voltage is high enough, there will be a high enough electric field between the bending streamer and the cathode plate for new streamer branches to emerge. These will propagate toward the cathode plate roughly following the background electric field lines.

We would like to emphasise the remarkable observation that the thick and thin streamers both propagate with the same velocity. Across the knotwilg branching there is a clear reduction in streamer thickness, but the discharge propagation velocity does not change.

The thick bending streamers seem to follow some invisible surface around the tip after having made the sharp turn. It is seen more often that streamers can follow special paths, e.g. those pre-ionised by previous streamers. We have found that at repetition rates of $14 \mathrm{~Hz}$ and higher subsequent streamers follow the same path (see section 3.1.1); similar to the time found in [21]. This repetition rate is an order of magnitude higher than the $1 \mathrm{~Hz}$ at which the knotwilg structures start to appear, but the streamer attracting regions are also much smaller, so the two observations could be consistent if the mechanism is dominated by electron diffusion.

Recently, there have been more observations of streamers following special paths. In [28] air streamers were found to follow the edge of an ionisation region created by a preceding different polarity inception cloud. In [21] streamers followed the edge of streamer channels from the previous discharge. And in [29] nitrogen streamers followed an ionised channel created by laser illumination. In these discharges the electrons are created respectively $100 \mathrm{~ns}, 100 \mu \mathrm{s}$ and maximally $2 \mathrm{~ms}$ before the streamer propagation. This is much shorter than in the case of knotwilg branching streamers, where there can be up to a second between discharges.

The presence of enough leftover background electrons or other leftover species is a requirement for their appearance. This is concluded from the fact that the knotwilg branches only appear at repetition rates of around $1 \mathrm{~Hz}$ and higher (see section 3.1). Therefore the leftover background electrons seem a likely candidate for the creation of the ionisation region for the streamers to propagate over.

Streamers are thick and smooth before the knotwilg branching and thin and with many branches after. This also hints toward different electron densities causing the knotwilg structures. Higher background electron densities were shown in previous research to thicken the streamer channel, and to suppress its branching [15, 19].

The localisation of leftover ionisation has not been measured directly. An estimate and to would have to include processes such as drift, diffusion and the post discharge chemistry, attachment and recombination as elaborated in $[20,21$, $23,29]$ and the streamers. We have found no indication for the presence of a sharp leftover electron density gradient (see section 3.1.1). Furthermore we expect any sharp gradient to be damped by diffusion between the electron creation and the appearance of the knotwilg branches (which is in the order of centimeters [20]). Therefore it is unlikely that there is a sharp electron density gradient over which the streamers propagate.

An alternative explanation is that the streamers follow the ionisation region at a specific critical electron density. Such an 
electron density has recently been found in pulsed microwave discharges [22]. No indications have been found in literature for the existence of a similar critical density in streamer discharges. Further experimental and modeling investigations of this question can deepen our understanding of streamer development.

\section{References}

[1] Ebert U, Montijn C, Briels T M P, Hundsdorfer W, Meulenbroek B, Rocco A and van Veldhuizen E M 2006 The multiscale nature of streamers Plasma Sources Sci. Technol. 15 S118

[2] Bazelyan E M and Raizer Y P 1998 Spark discharge (Boca Raton, FL: CRC)

[3] Gallimberti I, Bacchiega G, Bondiou-Clergerie A and Lalande P 2002 Fundamental processes in long air gap discharges C. R. Phys. 3 1335-59

[4] Bazelyan E M and Raizer Y P 2000 Lightning Physics and Lightning Protection (Bristol: Institute of Physics Publishing)

[5] Williams E R 2006 Problems in lightning physics-the role of polarity asymmetry Plasma Sources Sci. Technol. 15 S91

[6] Pasko V P 2007 Red sprite discharges in the atmosphere at high altitude: the molecular physics and the similarity with laboratory discharges Plasma Sources Sci. Technol. 16 S13

[7] Ebert U, Nijdam S, Li C, Luque A, Briels T and van Veldhuizen E 2010 Review of recent results on streamer discharges and discussion of their relevance for sprites and lightning J. Geophys. Res. 115 A00E43

[8] Clements J S, Mizuno A, Finney W C and Davis R H 1989 Combined removal of $\mathrm{SO}_{2}, \mathrm{NO}_{x}$, and fly ash from simulated flue gas using pulsed streamer corona IEEE Trans. Ind. Appl. 25 62-9

[9] van Veldhuizen E M 2000 Electrical Discharges for Environmental Purposes: Fundamentals and Applications (New York: Nova Science Publishers)

[10] Grabowski L, van Veldhuizen E, Pemen A and Rutgers W 2006 Corona above water reactor for systematic study of aqueous phenol degradation Plasma Chem. Plasma Process. 26 3-17

[11] Winands G J J, Yan K, Pemen A J M, Nair S A, Liu Z and van Heesch E J M 2006 An industrial streamer corona plasma system for gas cleaning IEEE Trans. Plasma Sci. 34 2426-33

[12] Kogelschatz U 2004 Atmospheric-pressure plasma technology Plasma Phys. Control. Fusion 46 B63

[13] Moreau E 2007 Airflow control by non-thermal plasma actuators J. Phys. D: Appl. Phys. 40605

[14] Starikovskii A Y, Anikin N B, Kosarev I N, Mintoussov E I, Nudnova M M, Rakitin A E, Roupassov D V, Starikovskaia S M and Zhukov V P 2008 Nanosecondpulsed discharges for plasma-assisted combustion and aerodynamics J. Propul. Power 241182
[15] Clevis T T J, Nijdam S and Ebert U 2013 Inception and propagation of positive streamers in high-purity nitrogen: effects of the voltage rise J. Phys. D: Appl. Phys. 46045202

[16] Briels T M P, Kos J, Winands G J J, van Veldhuizen E M and Ebert U 2008 Positive and negative streamers in ambient air: measuring diameter, velocity and dissipated energy J. Phys. D: Appl. Phys. 41234004

[17] Nijdam S, van de Wetering F M J H, Blanc R, van Veldhuizen E M and Ebert U 2010 Probing photo-ionization: experiments on positive streamers in pure gases and mixtures J. Phys. D: Appl. Phys. 43145204

[18] Briels T M P, Kos J, van Veldhuizen E M and Ebert U 2006 Circuit dependence of the diameter of pulsed positive streamers in air J. Phys. D: Appl. Phys. 395201

[19] Takahashi E, Kato S, Sasaki A, Kishimoto Y and Furutani H 2011 Controlling branching in streamer discharge by laser background ionization J. Phys. D: Appl. Phys. 44075204

[20] Nijdam S, Wormeester G, van Veldhuizen E M and Ebert U 2011 Probing background ionization: positive streamers with varying pulse repetition rate and with a radioactive admixture J. Phys. D: Appl. Phys. 44455201

[21] Nijdam S, Takahashi E, Markosyan A H and Ebert U 2014 Investigation of positive streamers by double-pulse experiments, effects of repetition rate and gas mixture Plasma Sources Sci. Technol. 23025008

[22] Carbone E and Nijdam S 2014 Ultra-fast pulsed microwave plasma breakdown: evidence of various ignition modes Plasma Sources Sci. Technol. 23012001

[23] Wormeester G, Pancheshnyi S, Luque A, Nijdam S and Ebert U 2010 Probing photo-ionization: simulations of positive streamers in varying $\mathrm{N}_{2}: \mathrm{O}_{2}$-mixtures $J$. Phys. $D$ : Appl. Phys. $\mathbf{4 3} 505201$

[24] Kossyi I A, Kostinsky A Y, Matveyev A A and Silakov V P 1992 Kinetic scheme of the non-equilibrium discharge in nitrogen-oxygen mixtures Plasma Sources Sci. Technol. 1207

[25] Grabowski L R, Briels T M P, van Veldhuizen E M and Pemen A J M 2005 Streamers in pulsed positive corona: low and high current regimes Proc. of the 27th ICPIG Conf. (Eindhoven) vol $27 \mathrm{pp}$ 04-425

[26] Chen S, Heijmans L C J, Zeng R, Nijdam S and Ebert U 2015 Nanosecond repetitively pulsed discharges in $\mathrm{N}_{2}-\mathrm{O}_{2}$ mixtures: inception cloud and streamer emergence $J$. Phys. D: Appl. Phys. 48175201

[27] Wormeester G, Nijdam S and Ebert U 2011 Feather-like structures in positive streamers interpreted as electron avalanches Japan. J. Appl. Phys. 50 08JA01

[28] Nijdam S, Miermans K, Van E M and Ebert U 2011 A peculiar streamer morphology created by a complex voltage pulse IEEE Trans. Plasma Sci. 39 2216-7

[29] Nijdam S, Takahashi E, Teunissen J and Ebert U 2014 Streamer discharges can move perpendicularly to the electric field New J. Phys. 16103038 